\title{
Real method of interpolation on subcouples of codimension one
}

\author{
by \\ S. V. Astashkin (Samara) and P. Sunehag (Canberra)
}

\begin{abstract}
We find necessary and sufficient conditions under which the norms of the interpolation spaces $\left(N_{0}, N_{1}\right)_{\theta, q}$ and $\left(X_{0}, X_{1}\right)_{\theta, q}$ are equivalent on $N$, where $N$ is the kernel of a nonzero functional $\psi \in\left(X_{0} \cap X_{1}\right)^{*}$ and $N_{i}$ is the normed space $N$ with the norm inherited from $X_{i}(i=0,1)$. Our proof is based on reducing the problem to its partial case studied by Ivanov and Kalton, where $\psi$ is bounded on one of the endpoint spaces. As an application we completely resolve the problem of when the range of the operator $T_{\theta}=S-2^{\theta} I$ ( $S$ denotes the shift operator and $I$ the identity) is closed in any $\ell_{p}(\mu)$, where the weight $\mu=\left(\mu_{n}\right)_{n \in \mathbb{Z}}$ satisfies the inequalities $\mu_{n} \leq \mu_{n+1} \leq 2 \mu_{n}(n \in \mathbb{Z})$.
\end{abstract}

1. Introduction. Interpolation of subspaces is an important and rather complicated problem in interpolation theory [5]. It has been stated as early as in the monograph [12] by Lions and Magenes. Various aspects of this problem have been treated in $[1,6-9,13-15,18,19]$ (the authors are very far from claiming the completeness of this citation list). Here, we shall consider a partial case of this general subspace interpolation problem, interpolation of intersections by the real method.

Let $\left(X_{0}, X_{1}\right)$ be a Banach couple, i.e., a pair of Banach spaces linearly and continuously embedded in a Hausdorff topological vector space $\tau$. Every linear subspace $N$ of $\tau$ generates a normed (in general, non-Banach) couple $\left(X_{0} \cap N, X_{1} \cap N\right)$, where the norm on $X_{i} \cap N$ is just obtained by the restriction of the norm of $X_{i}(i=0,1)$.

The problem of interpolation of intersections is the problem of finding conditions on the triple $\left(X_{0}, X_{1}, N\right)$ and the parameters $\theta \in(0,1)$ and $q \in$ $[1, \infty]$ of the real interpolation method under which the natural formula (with equivalence of norms)

$$
\left(X_{0} \cap N, X_{1} \cap N\right)_{\theta, q}=\left(X_{0}, X_{1}\right)_{\theta, q} \cap N
$$

is valid.

2000 Mathematics Subject Classification: Primary 46M35; Secondary 46E30, 46A45.

Key words and phrases: interpolation, real method of interpolation, subcouple, weighted spaces, shift operator, spectrum. 
Note that when $N$ is the kernel of a linear functional $\psi \in\left(X_{0} \cap X_{1}\right)^{*}$ formula (1) means just the equivalence of the norms of the spaces $N_{\theta, q}=$ $\left(X_{0} \cap N, X_{1} \cap N\right)_{\theta, q}$ and $X_{\theta, q}=\left(X_{0}, X_{1}\right)_{\theta, q}$ on the subspace $N$. In [2, 3] and independently in [17] four dilation indices of the Peetre $K$-functional $K\left(t, \psi ; X_{0}^{*}, X_{1}^{*}\right)$ in the dual couple $\left(X_{0}^{*}, X_{1}^{*}\right)$ have been introduced and the problem has been solved under an extra condition on these indices. Here we shall give a solution of this problem without any additional conditions.

An important particular case of the problem on the interpolation of intersections generated by a linear functional was treated in [11]. Namely, sufficient conditions on $\theta \in(0,1), p \in[1, \infty)$, and on weight functions $w_{0}(x)$ and $w_{1}(x)$ were found ensuring the formula

$$
\left(L_{p}\left(w_{0}\right) \cap N, L_{p}\left(w_{1}\right) \cap N\right)_{\theta, p}=\left(L_{p}\left(w_{0}\right), L_{p}\left(w_{1}\right)\right)_{\theta, p} \cap N,
$$

where $L_{p}(w)$ is a weighted $L_{p^{-}}$space on $(0, \infty)$ with the usual norm and $N$ is the space of all functions $f:(0, \infty) \rightarrow \mathbb{R}$ satisfying

$$
\int_{0}^{\infty} f(x) d x=0 .
$$

It turned out that the validity of (2) is closely related to the possibility of "interpolating" certain Hardy-type integral inequalities. In the same paper [11], the following more general question was asked: under what conditions on $w_{0}(x), w_{1}(x), p_{0}, p_{1} \in[1, \infty), \theta \in(0,1)$, and $q \in[1, \infty]$ do we have the identity

$$
\left(L_{p_{0}}\left(w_{0}\right) \cap N, L_{p_{1}}\left(w_{1}\right) \cap N\right)_{\theta, q}=\left(L_{p_{0}}\left(w_{0}\right), L_{p_{1}}\left(w_{1}\right)\right)_{\theta, q} \cap N ?
$$

(Generally speaking, here $p_{0} \neq p_{1}$, and $N$ is still determined by (3).) We note that our main results (Theorems 1 and 2) imply a solution of this problem. Not entering into details, we will concentrate here only on the case of $L_{1}$-spaces (for more detailed consideration of this topic see [3]).

In [6], a connection was discovered between interpolation of subspaces and the problem of determining if the range of the operator $T_{\theta}=S-2^{\theta} I$ on weighted $\ell_{p}$-spaces is closed or not. Here, $S$ denotes the shift operator and $I$ the identity. We will completely resolve this problem in the case when the weight $\mu=\left(\mu_{n}\right)_{n \in \mathbb{Z}}$ satisfies the inequalities $\mu_{n} \leq \mu_{n+1} \leq 2 \mu_{n}(n \in \mathbb{Z})$.

2. Definitions, notation, and auxiliary results. For an arbitrary normed couple $\left(X_{0}, X_{1}\right)$ and $t>0$ we define the Peetre $K$-functional:

$$
K\left(t, x ; X_{0}, X_{1}\right)=\inf _{x=x_{0}+x_{1}, x_{i} \in X_{i}}\left(\left\|x_{0}\right\|_{X_{0}}+t\left\|x_{1}\right\|_{X_{1}}\right), \quad x \in X_{0}+X_{1} .
$$


If $0<\theta<1$ and $1 \leq q<\infty$, then the real interpolation space $X_{\theta, q}=$ $\left(X_{0}, X_{1}\right)_{\theta, q}$ consists of all $x \in X_{0}+X_{1}$ such that

$$
\|x\|_{X_{\theta, q}}=\left\{\int_{0}^{\infty}\left(t^{-\theta} K\left(t, x ; X_{0}, X_{1}\right)\right)^{q} \frac{d t}{t}\right\}^{1 / q}<\infty .
$$

Analyzing the proof of the equivalence theorem [5, Theorem 3.3.1], we see that it remains true for normed (and not only Banach) couples. Thus, the space $X_{\theta, q}$ (with an equivalent norm) can be defined in terms of the $J$ functional

$$
J\left(t, x ; X_{0}, X_{1}\right)=\max \left(\|x\|_{X_{0}}, t\|x\|_{X_{1}}\right), \quad x \in X_{0} \cap X_{1} .
$$

Specifically, $X_{\theta, q}$ consists of all $x \in X_{0}+X_{1}$ representable in the form

$$
x=\sum_{k \in \mathbb{Z}} 2^{\theta k} x_{k} \quad \text { (convergence in } X_{0}+X_{1} \text { ) }
$$

with the norm

$$
\inf \left\{\sum_{k \in \mathbb{Z}}\left(J\left(2^{k}, x_{k} ; X_{0}, X_{1}\right)\right)^{q}\right\}^{1 / q},
$$

where the infimum is taken over all representations indicated above.

We will say that a Banach couple $\left(X_{0}, X_{1}\right)$ is regular if $X_{0} \cap X_{1}$ is dense in both $X_{0}$ and $X_{1}$. If $\left(X_{0}, X_{1}\right)$ is regular it follows that $X_{0}^{*}$ and $X_{1}^{*}$ are naturally embedded into $\left(X_{0} \cap X_{1}\right)^{*}$, and so we may consider the Banach couple $\left(X_{0}^{*}, X_{1}^{*}\right)$ of the dual spaces. Moreover, $\left(X_{0} \cap X_{1}\right)^{*}=X_{0}^{*}+X_{1}^{*}[5$, Theorem 2.7.1].

Suppose that $\left(X_{0}, X_{1}\right)$ is a regular Banach couple and $\psi \in\left(X_{0} \cap X_{1}\right)^{*}$. Then $\psi \in X_{0}^{*}+X_{1}^{*}$. In what follows, the $K$-functional $k(t)=K\left(t, \psi ; X_{0}^{*}, X_{1}^{*}\right)$ will play a crucial role. Define the functions

$$
\begin{gathered}
M(t)=\sup _{s>0} \frac{k(t s)}{k(s)}, \quad M_{0}(t)=\sup _{0<s \leq \min (1,1 / t)} \frac{k(t s)}{k(s)}, \\
M_{\infty}(t)=\sup _{s \geq \max (1,1 / t)} \frac{k(t s)}{k(s)} .
\end{gathered}
$$

They are submultiplicative for $t>0$, and therefore the following numbers are well defined:

$$
\begin{aligned}
& \alpha=\lim _{t \rightarrow 0} \frac{\log _{2} M(t)}{\log _{2} t}, \quad \alpha_{0}=\lim _{t \rightarrow 0} \frac{\log _{2} M_{0}(t)}{\log _{2} t}, \quad \alpha_{\infty}=\lim _{t \rightarrow 0} \frac{\log _{2} M_{\infty}(t)}{\log _{2} t}, \\
& \beta=\lim _{t \rightarrow \infty} \frac{\log _{2} M(t)}{\log _{2} t}, \quad \beta_{0}=\lim _{t \rightarrow \infty} \frac{\log _{2} M_{0}(t)}{\log _{2} t}, \quad \beta_{\infty}=\lim _{t \rightarrow \infty} \frac{\log _{2} M_{\infty}(t)}{\log _{2} t},
\end{aligned}
$$

which are called the dilation indices of $k(t)$. It is easily seen that $0 \leq \alpha \leq$ $\alpha_{0} \leq \beta_{0} \leq \beta \leq 1$ and $0 \leq \alpha \leq \alpha_{\infty} \leq \beta_{\infty} \leq \beta \leq 1$. In [3] and [17] only four indices were introduced and even though we will here use six indices, in 
our formulations they in fact reduce to four, since the relationship presented below holds.

LEMMA 1. We have

$$
\alpha=\min \left(\alpha_{0}, \alpha_{\infty}\right), \quad \beta=\max \left(\beta_{0}, \beta_{\infty}\right) .
$$

Proof. These equalities have very similar proofs and we give only the proof for the $\beta$ part. First, the following formula is true:

$$
\frac{\log _{2}\left(\frac{k(t s)}{k(s)}\right)}{\log _{2} t}=(1-\lambda) \frac{\log _{2}\left(\frac{k\left(t^{1-\lambda}\right)}{k(1)}\right)}{\log _{2}\left(t^{1-\lambda}\right)}+\lambda \frac{\log _{2}\left(\frac{k\left(t^{\lambda} t^{-\lambda}\right)}{k\left(t^{-\lambda}\right)}\right)}{\log _{2}\left(t^{\lambda}\right)},
$$

where $t>1, \lambda \in(0,1)$, and $s=1 / t^{\lambda}$. In fact,

$$
\begin{aligned}
\frac{\log _{2}\left(\frac{k(t s)}{k(s)}\right)}{\log _{2} t} & =\frac{\log _{2}\left(\frac{k(t s)}{k(1)}\right)+\log _{2}\left(\frac{k(1)}{k(s)}\right)}{\log _{2} t} \\
& =(1-\lambda) \frac{\log _{2}\left(\frac{k\left(t^{1-\lambda}\right)}{k(1)}\right)}{\log _{2}\left(t^{1-\lambda}\right)}+\lambda \frac{\log _{2}\left(\frac{k\left(t^{\lambda} t^{-\lambda}\right)}{k\left(t^{-\lambda}\right)}\right)}{\log _{2}\left(t^{\lambda}\right)},
\end{aligned}
$$

and (6) is proved.

It is clear that the claim will be proved if we show that

$$
\lim _{t \rightarrow \infty} \frac{\log _{2}\left(\sup _{s>0} \frac{k(t s)}{k(s)}\right)}{\log _{2} t}=\lim _{t \rightarrow \infty} \frac{\log _{2}\left(\sup _{s \notin(1 / t, 1)} \frac{k(t s)}{k(s)}\right)}{\log _{2} t} .
$$

For every $t>1$ choose $s(t) \in[1 / t, 1]$ such that

$$
\sup _{s \in[1 / t, 1]} \frac{k(t s)}{k(s)}=\frac{k(t s(t))}{k(s(t))} .
$$

Then $s(t)=t^{-\lambda(t)}$, where $0 \leq \lambda(t) \leq 1$. If there is a sequence $t_{n}>0$ such that $t_{n} \rightarrow \infty, t_{n}^{\lambda\left(t_{n}\right)} \rightarrow \infty$, and $t_{n}^{1-\lambda\left(t_{n}\right)} \rightarrow \infty$ as $n \rightarrow \infty$, then the result follows from formula (6). If there is no sequence $\left(t_{n}\right)$ such that $t_{n} \rightarrow \infty$ and $t_{n}^{\lambda\left(t_{n}\right)} \rightarrow \infty$, then $\lambda(t) \rightarrow 0$ as $t \rightarrow \infty$. In such a case we can use (6) again to reach our result, and likewise if there is no sequence $\left(t_{n}\right)$ such that $t_{n} \rightarrow \infty$ and $t_{n}^{1-\lambda\left(t_{n}\right)} \rightarrow \infty$ since then $\lambda(t) \rightarrow 1$ as $t \rightarrow \infty$.

The second lemma will be useful for reducing our problem to the case when a functional is bounded on one of the endpoint spaces.

Lemma 2. If $\psi \in\left(X_{0} \cap X_{1}\right)^{*}$ then

$$
\begin{aligned}
\alpha_{0}\left(\psi,\left(X_{0} \cap X_{1}, X_{1}\right)\right) & =\alpha_{0}\left(\psi,\left(X_{0}, X_{1}\right)\right), \\
\beta_{0}\left(\psi,\left(X_{0} \cap X_{1}, X_{1}\right)\right) & =\beta_{0}\left(\psi,\left(X_{0}, X_{1}\right)\right), \\
\alpha_{\infty}\left(\psi,\left(X_{0} \cap X_{1}, X_{1}\right)\right) & =\alpha\left(\psi,\left(X_{0} \cap X_{1}, X_{1}\right)\right)=0, \\
\beta_{\infty}\left(\psi,\left(X_{0} \cap X_{1}, X_{1}\right)\right) & =0
\end{aligned}
$$


and

$$
\begin{aligned}
\alpha_{\infty}\left(\psi,\left(X_{0}, X_{0} \cap X_{1}\right)\right) & =\alpha_{\infty}\left(\psi,\left(X_{0}, X_{1}\right)\right), \\
\beta_{\infty}\left(\psi,\left(X_{0}, X_{0} \cap X_{1}\right)\right) & =\beta_{\infty}\left(\psi,\left(X_{0}, X_{1}\right)\right), \\
\beta_{0}\left(\psi,\left(X_{0}, X_{0} \cap X_{1}\right)\right) & =\beta\left(\psi,\left(X_{0}, X_{0} \cap X_{1}\right)\right)=1, \\
\alpha_{0}\left(\psi,\left(X_{0}, X_{0} \cap X_{1}\right)\right) & =1 .
\end{aligned}
$$

Proof. It is true that

$$
K\left(t, \psi ; X_{0}^{*}, X_{1}^{*}\right)= \begin{cases}K\left(t, \psi ; X_{0}^{*},\left(X_{0} \cap X_{1}\right)^{*}\right) & \text { if } t \geq 1 \\ K\left(t, \psi ;\left(X_{0} \cap X_{1}\right)^{*}, X_{1}^{*}\right) & \text { if } t \leq 1\end{cases}
$$

Also notice that since $\psi \in\left(X_{0} \cap X_{1}\right)^{*}$ we know that $k_{1}(t):=K(t, \psi$; $\left.\left(X_{0} \cap X_{1}\right)^{*}, X_{1}^{*}\right)$ is bounded from above and the same is true for $k_{0}(t) / t$, where $k_{0}(t):=K\left(t, \psi ; X_{0},\left(X_{0} \cap X_{1}\right)^{*}\right)$. The results follow from these properties.

3. Main results. Suppose that $\left(X_{0}, X_{1}\right)$ is a regular Banach couple. Let $\psi \in\left(X_{0} \cap X_{1}\right)^{*}$ be a nonzero functional, let $N=\operatorname{ker} \psi$, and let $N_{i}$ be the normed space $N$ with the norm inherited from $X_{i}(i=0,1)$.

THEOREM 1. The norms of the interpolation spaces $N_{\theta, q}=\left(N_{0}, N_{1}\right)_{\theta, q}$ and $X_{\theta, q}=\left(X_{0}, X_{1}\right)_{\theta, q}$ are equivalent on $N$ (i.e., $\left.N_{\theta, q}=X_{\theta, q} \cap N\right)$ if and only if

$$
\theta \in(0, \alpha) \cup\left(\beta_{\infty}, \alpha_{0}\right) \cup\left(\beta_{0}, \alpha_{\infty}\right) \cup(\beta, 1) .
$$

Moreover, if $\theta \in(0, \alpha) \cup\left(\beta_{0}, \alpha_{\infty}\right) \cup(\beta, 1)$, then $N_{\theta, q}$ is dense in $X_{\theta, q}$; if $\theta \in\left(\beta_{\infty}, \alpha_{0}\right)$, then $N_{\theta, q}$ is dense in some subspace of $X_{\theta, q}$ of codimension 1.

Using the following definition from [17], we can state Theorem 1 in another way. As above, $\left(X_{0}, X_{1}\right)$ is a regular Banach couple, $\psi \in\left(X_{0} \cap X_{1}\right)^{*}$, and $N=\operatorname{ker} \psi$. For arbitrary $0<\theta<1$ and $1 \leq q<\infty$, we denote by $X_{\theta, q, \psi}=\left(X_{0}, X_{1}\right)_{\theta, q, \psi}$ the set of all $x \in X_{0}+X_{1}$ representable in the form

$$
x=\sum_{k \in \mathbb{Z}} 2^{\theta k} x_{k}, \quad x_{k} \in N \quad \text { (convergence in } X_{0}+X_{1} \text { ) }
$$

with the norm (5), where the infimum is taken over all representations of the form (8).

THEOREM 2. The space $X_{\theta, q, \psi}$ is closed in the real interpolation space $X_{\theta, q}=\left(X_{0}, X_{1}\right)_{\theta, q}$ if and only if condition (7) holds. Moreover, if $\theta \in(0, \alpha) \cup$ $\left(\beta_{0}, \alpha_{\infty}\right) \cup(\beta, 1)$, then $X_{\theta, q, \psi}=X_{\theta, q}$; if $\theta \in\left(\beta_{\infty}, \alpha_{0}\right)$, then $X_{\theta, q, \psi}=X_{\theta, q} \cap$ ker $\widetilde{\psi}$, where the functional $\widetilde{\psi}$ is a continuous extension of $\psi$ to the space $X_{\theta, q}$.

REMARK 1. In what follows, the interval $(\alpha, \beta)$, as usual, is the set of all reals $x$ satisfying the inequality $\alpha<x<\beta$. Thus, the inequalities $\alpha_{0} \leq \beta_{0}$ 
and $\alpha_{\infty} \leq \beta_{\infty}$ imply that at most one of the intervals $\left(\beta_{0}, \alpha_{\infty}\right)$ and $\left(\beta_{\infty}, \alpha_{0}\right)$ may be nonempty.

REMARK 2. Theorems 1 and 2 strengthen Theorem 1 from [3] and Proposition 5.6 from [17], which contain the extra condition $\beta_{\infty} \leq \alpha_{0}$.

4. Proofs: Reduction to the Ivanov-Kalton case. Our proof of the main results is based on reducing the problem to two problems of the kind studied by Ivanov and Kalton in [6], i.e., to a case where we have a linear functional that is bounded on one of the endpoint spaces.

Suppose that we have a Banach couple $\bar{X}=\left(X_{0}, X_{1}\right)$ and a linear functional $\psi \in\left(X_{0} \cap X_{1}\right)^{*}$. Suppose also that $X$ is an intermediate space for $\left(X_{0}, X_{1}\right)$ and that $\psi$ can be extended to a bounded linear functional on $X$. Then we will write $\psi \in X^{*}$, which is meant to imply that we have extended $\psi$ to $X$. For every $\psi \in\left(X_{0} \cap X_{1}\right)^{*}$ there is a minimal interpolation space norm for which $\psi$ is bounded. It is unique up to equivalence. That norm is

$$
\|x\|_{\psi}=\sup _{\|T\|_{\bar{X} \rightarrow \bar{X} \leq 1}}|\langle\psi, T x\rangle| .
$$

Whenever we write ker $\psi$ in this section, we assume that $\psi$ has been extended to the corresponding maximal interpolation space for which $\psi$ is bounded.

THEOREM 3. Suppose that $\bar{X}=\left(X_{0}, X_{1}\right)$ is a regular Banach couple, that $\psi \in\left(X_{0} \cap X_{1}\right)^{*}$ and that $\psi \neq 0$. Then $\left(X_{0}, X_{1}\right)_{\theta, q, \psi}$ is a closed subspace of the interpolation space $\left(X_{0}, X_{1}\right)_{\theta, q}$ if and only if both $\left(X_{0}, X_{0} \cap X_{1} \cap \operatorname{ker} \psi\right)_{\theta, q}$ is a closed subspace of $\left(X_{0}, X_{0} \cap X_{1}\right)_{\theta, q}$ and $\left(X_{0} \cap X_{1} \cap \operatorname{ker} \psi, X_{1}\right)_{\theta, q}$ is a closed subspace of $\left(X_{0} \cap X_{1}, X_{1}\right)_{\theta, q}$. Moreover:

(i) The formula

$$
\left(X_{0}, X_{1}\right)_{\theta, q, \psi}=\left(X_{0}, X_{1}\right)_{\theta, q} \cap \operatorname{ker} \psi
$$

is valid if and only if both

$$
\left(X_{0}, X_{0} \cap X_{1} \cap \operatorname{ker} \psi\right)_{\theta, q}=\left(X_{0}, X_{0} \cap X_{1}\right)_{\theta, q} \cap \operatorname{ker} \psi
$$

and

$$
\left(X_{0} \cap X_{1} \cap \operatorname{ker} \psi, X_{1}\right)_{\theta, q}=\left(X_{0} \cap X_{1}, X_{1}\right)_{\theta, q} \cap \operatorname{ker} \psi .
$$

(ii) The formula

$$
\left(X_{0}, X_{1}\right)_{\theta, q, \psi}=\left(X_{0}, X_{1}\right)_{\theta, q}
$$

is valid if and only if both

$$
\left(X_{0}, X_{0} \cap X_{1} \cap \operatorname{ker} \psi\right)_{\theta, q}=\left(X_{0}, X_{0} \cap X_{1}\right)_{\theta, q}
$$

and

$$
\left(X_{0} \cap X_{1} \cap \operatorname{ker} \psi, X_{1}\right)_{\theta, q}=\left(X_{0} \cap X_{1}, X_{1}\right)_{\theta, q}
$$


or both

$$
\left(X_{0}, X_{0} \cap X_{1} \cap \operatorname{ker} \psi\right)_{\theta, q}=\left(X_{0}, X_{0} \cap X_{1}\right)_{\theta, q}
$$

and

$\left(X_{0} \cap X_{1} \cap \operatorname{ker} \psi, X_{1}\right)_{\theta, q}=\left(X_{0} \cap X_{1}, X_{1}\right)_{\theta, q} \cap \operatorname{ker} \psi$,

or both

$$
\left(X_{0}, X_{0} \cap X_{1} \cap \operatorname{ker} \psi\right)_{\theta, q}=\left(X_{0}, X_{0} \cap X_{1}\right)_{\theta, q} \cap \operatorname{ker} \psi
$$

and

$$
\left(X_{0} \cap X_{1} \cap \operatorname{ker} \psi, X_{1}\right)_{\theta, q}=\left(X_{0} \cap X_{1}, X_{1}\right)_{\theta, q} .
$$

For the proof of the theorem we need four lemmas.

LEMMA 3.

$$
\begin{aligned}
\left(X_{0}, X_{1}\right)_{\theta, q} & =\left(X_{0}, X_{1}\right)_{\theta, q} \cap X_{0}+\left(X_{0}, X_{1}\right)_{\theta, q} \cap X_{1}, \\
\left(X_{0}, X_{1}\right)_{\theta, q, \psi} & =\left(X_{0}, X_{1}\right)_{\theta, q, \psi} \cap X_{0}+\left(X_{0}, X_{1}\right)_{\theta, q, \psi} \cap X_{1} .
\end{aligned}
$$

Proof. It is clear that

$$
\left(X_{0}, X_{1}\right)_{\theta, q} \cap X_{0}+\left(X_{0}, X_{1}\right)_{\theta, q} \cap X_{1} \subset\left(X_{0}, X_{1}\right)_{\theta, q}
$$

since the latter space is a vector space.

Assume that $x \in\left(X_{0}, X_{1}\right)_{\theta, q}$. Then there are $x_{k} \in X_{0} \cap X_{1}$ such that $x=\sum x_{k} 2^{\theta k}$ and $\left\|\left(J\left(2^{k}, x_{k} ; X_{0}, X_{1}\right)\right)\right\|_{q}<\infty$. We now want to find $x_{0} \in$ $\left(X_{0}, X_{1}\right)_{\theta, q} \cap X_{0}$ and $x_{1} \in\left(X_{0}, X_{1}\right)_{\theta, q} \cap X_{1}$ such that $x=x_{0}+x_{1}$. Let $x_{0}=\sum_{k=-\infty}^{0} x_{k} 2^{\theta k}, x_{1}=\sum_{k=1}^{\infty} x_{k} 2^{\theta k}$. The convergence of these two series in $X_{0}$ and $X_{1}$ follows from the facts that $\sum_{k=-\infty}^{0}\left\|2^{\theta k} x_{k}\right\|_{X_{0}}<\infty$ and $\sum_{k=1}^{\infty}\left\|2^{\theta k} x_{k}\right\|_{X_{1}}<\infty$ and that $X_{0}$ and $X_{1}$ are Banach spaces.

The second claim has the same proof.

The following lemma is proved in [3] (see also [14]).

LEMMA 4.

$$
\begin{aligned}
& \left(X_{0}, X_{0} \cap X_{1}\right)_{\theta, q}=\left(X_{0}, X_{1}\right)_{\theta, q} \cap X_{0}, \\
& \left(X_{0} \cap X_{1}, X_{1}\right)_{\theta, q}=\left(X_{0}, X_{1}\right)_{\theta, q} \cap X_{1} .
\end{aligned}
$$

LEMMA 5.

(i) If $\psi \in\left(X_{0}, X_{0} \cap X_{1}\right)_{\theta, q}^{*}$, then

$$
\begin{aligned}
& \left(X_{0}, X_{0} \cap X_{1} \cap \operatorname{ker} \psi\right)_{\theta, q}=\left(X_{0}, X_{1}\right)_{\theta, q, \psi} \cap X_{0} \cap \operatorname{ker} \psi, \\
& \left(X_{0} \cap X_{1} \cap \operatorname{ker} \psi, X_{1}\right)_{\theta, q}=\left(X_{0}, X_{1}\right)_{\theta, q, \psi} \cap X_{1} .
\end{aligned}
$$

(ii) If $\psi \in\left(X_{0} \cap X_{1}, X_{1}\right)_{\theta, q}^{*}$, then

$$
\begin{aligned}
& \left(X_{0} \cap X_{1} \cap \operatorname{ker} \psi, X_{1}\right)_{\theta, q}=\left(X_{0}, X_{1}\right)_{\theta, q, \psi} \cap X_{1} \cap \operatorname{ker} \psi, \\
& \left(X_{0}, X_{0} \cap X_{1} \cap \operatorname{ker} \psi\right)_{\theta, q}=\left(X_{0}, X_{1}\right)_{\theta, q, \psi} \cap X_{0} .
\end{aligned}
$$


(iii) If $\psi \notin\left(X_{0}, X_{0} \cap X_{1}\right)_{\theta, q}^{*}$ and $\left(X_{0}, X_{1}\right)_{\theta, q}=\left(X_{0}, X_{1}\right)_{\theta, q, \psi}$, then $\left(X_{0}, X_{0} \cap X_{1} \cap \operatorname{ker} \psi\right)_{\theta, q}=\left(X_{0}, X_{1}\right)_{\theta, q, \psi} \cap X_{0}$.

(iv) If $\psi \notin\left(X_{0} \cap X_{1}, X_{1}\right)_{\theta, q}^{*}$ and $\left(X_{0}, X_{1}\right)_{\theta, q}=\left(X_{0}, X_{1}\right)_{\theta, q, \psi}$, then $\left(X_{0} \cap X_{1} \cap \operatorname{ker} \psi, X_{1}\right)_{\theta, q}=\left(X_{0}, X_{1}\right)_{\theta, q, \psi} \cap X_{1}$.

Proof. Assume that $\psi \in\left(X_{0}, X_{0} \cap X_{1}\right)_{\theta, q}^{*}$ and that $x \in\left(X_{0}, X_{1}\right)_{\theta, q, \psi} \cap$ $X_{0} \cap \operatorname{ker} \psi$. Then there are $x_{k} \in X_{0} \cap X_{1} \cap \operatorname{ker} \psi$ such that $x=\sum 2^{\theta k} x_{k}$ with convergence in the $X_{0}+X_{1}$ norm and $\left\|\left(J\left(2^{k}, x_{k} ; X_{0}, X_{1}\right)\right)\right\|_{q}<\infty$. It is readily seen that $J\left(2^{k}, x_{k} ; X_{0}, X_{0} \cap X_{1} \cap \operatorname{ker} \psi\right)=J\left(2^{k}, x_{k} ; X_{0}, X_{1}\right)$ if $k \leq 0$. Therefore, the series $u=\sum_{k=-\infty}^{-1} 2^{\theta k} x_{k}$ converges in the norm of $\left(X_{0}, X_{0} \cap X_{1}\right)_{\theta, q}$, and hence $\psi(u)=0$. Since $x \in X_{0} \cap \operatorname{ker} \psi$, we see that $s=x-u=\sum_{k=0}^{\infty} 2^{\theta k} x_{k} \in X_{0} \cap X_{1} \cap \operatorname{ker} \psi$. Let $y_{k}=x_{k}$ if $k<0, y_{0}=s$, and $y_{k}=0$ if $k>0$. Then $y_{k} \in X_{0} \cap X_{1} \cap \operatorname{ker} \psi,\left\|\left(J\left(2^{k}, y_{k} ; X_{0}, X_{0} \cap X_{1}\right)\right)\right\|_{q}<\infty$, and $\sum 2^{\theta k} y_{k}=x$ (convergence in $X_{0}$ ). Thus,

$$
\left(X_{0}, X_{1}\right)_{\theta, q, \psi} \cap X_{0} \cap \operatorname{ker} \psi \subset\left(X_{0}, X_{0} \cap X_{1} \cap \operatorname{ker} \psi\right)_{\theta, q} .
$$

From the assumption that $\psi \in\left(X_{0}, X_{0} \cap X_{1}\right)_{\theta, q}^{*}$ it follows that the opposite inclusion is valid as well, and the first formula from (i) is proved.

Next, assume that $\psi \in\left(X_{0} \cap X_{1}, X_{1}\right)_{\theta, q}^{*}$. We will use the same idea again. For each $x \in\left(X_{0}, X_{1}\right)_{\theta, q, \psi} \cap X_{0}$ there are $x_{k} \in X_{0} \cap X_{1} \cap \operatorname{ker} \psi$ such that $x=$ $\sum 2^{\theta k} x_{k}$ with convergence in the $X_{0}+X_{1}$ norm and $\left\|\left(J\left(2^{k}, x_{k} ; X_{0}, X_{1}\right)\right)\right\|_{q}$ $<\infty$. Again the series $\sum_{k=-\infty}^{-1} 2^{\theta k} x_{k}$ converges in $X_{0}$ and $s=\sum_{k=0}^{\infty} 2^{\theta k} x_{k} \in$ $X_{0} \cap X_{1}$. Moreover, $\psi(s)$ is well defined since $s \in X_{0} \cap X_{1}$. Note that $J\left(2^{k}, x_{k} ; X_{0} \cap X_{1} \cap \operatorname{ker} \psi, X_{1}\right)=J\left(2^{k}, x_{k} ; X_{0}, X_{1}\right)$ if $k \geq 0$. This implies that the series $s=\sum_{k=0}^{\infty} 2^{\theta k} x_{k}$ converges in the norm of $\left(X_{0} \cap X_{1}, X_{1}\right)_{\theta, q}$. Since $\psi \in\left(X_{0} \cap X_{1}, X_{1}\right)_{\theta, q}^{*}$, we have $\psi(s)=0$ and we can as above conclude that $x \in\left(X_{0}, X_{0} \cap X_{1} \cap \text { ker } \psi\right)_{\theta, q}$. Therefore,

$$
\left(X_{0}, X_{1}\right)_{\theta, q, \psi} \cap X_{0} \subset\left(X_{0}, X_{0} \cap X_{1} \cap \operatorname{ker} \psi\right)_{\theta, q} .
$$

Since the opposite inclusion is obvious the second formula of (ii) is proved.

Finally, we consider (iii). We keep previous arguments and notation. If $\psi \notin\left(X_{0} \cap X_{1}, X_{1}\right)_{\theta, q}^{*}$ it is possible that $\psi(s) \neq 0$. If there are elements $w_{k}$ in $X_{0} \cap X_{1} \cap \operatorname{ker} \psi$ such that $\sum 2^{\theta k} w_{k}=0,\left\|\left(J\left(2^{k}, w_{k} ; X_{0}, X_{1}\right)\right)\right\|_{q}<\infty$ and $\psi\left(\sum_{k=0}^{\infty} 2^{\theta k} w_{k}\right)=1$, then there is another representation $x=\sum 2^{\theta k} v_{k}$ such that $\left\|\left(J\left(2^{k}, v_{k} ; X_{0}, X_{1}\right)\right)\right\|_{q}<\infty$ and $\psi\left(\sum_{k=0}^{\infty} 2^{\theta k} v_{k}\right)=0$ and we can draw the desired conclusion.

To finish this proof we now assume that there are no such elements, i.e., $\psi\left(\sum_{k=0}^{\infty} 2^{\theta k} w_{k}\right)=0$ whenever $w_{k}$ are elements in $X_{0} \cap X_{1} \cap$ ker $\psi$ such that $\sum 2^{\theta k} w_{k}=0$ and $\left\|\left(J\left(2^{k}, w_{k} ; X_{0}, X_{1}\right)\right)\right\|_{q}<\infty$. In other words, if an element $x \in\left(X_{0}, X_{1}\right)_{\theta, q} \cap X_{0}$ has two representations:

$$
x=\sum 2^{\theta k} x_{k} \quad \text { and } \quad x=\sum 2^{\theta k} y_{k}
$$


such that

$$
\left\|\left(J\left(2^{k}, x_{k} ; X_{0}, X_{1}\right)\right)\right\|_{q}<\infty, \quad\left\|\left(J\left(2^{k}, y_{k} ; X_{0}, X_{1}\right)\right)\right\|_{q}<\infty,
$$

and $\left(x_{k}\right) \cup\left(y_{k}\right) \subset X_{0} \cap X_{1} \cap \operatorname{ker} \psi$, then

$$
\psi\left(\sum_{k=0}^{\infty} 2^{\theta k} x_{k}\right)=\psi\left(\sum_{k=0}^{\infty} 2^{\theta k} y_{k}\right) .
$$

Thus, we can define a linear functional $\phi$ on $\left(X_{0}, X_{1}\right)_{\theta, q} \cap X_{0}$. In fact, if $x \in\left(X_{0}, X_{1}\right)_{\theta, q} \cap X_{0}$, then by the extra assumption from part (iii) of the lemma, i.e., that $\left(X_{0}, X_{1}\right)_{\theta, q}=\left(X_{0}, X_{1}\right)_{\theta, q, \psi}$, we have $x=\sum_{k=-\infty}^{\infty} 2^{\theta k} x_{k}$, where $x_{k} \in X_{0} \cap X_{1} \cap \operatorname{ker} \psi$. Then we set

$$
\phi(x):=\psi\left(\sum_{k=0}^{\infty} 2^{\theta k} x_{k}\right) .
$$

If $x \in X_{0} \cap X_{1} \cap \operatorname{ker} \psi$, then $\phi(x)=0$, since we can represent $x$ as $\sum 2^{\theta k} x_{k}$ by letting $x_{0}=x$ and $x_{k}=0$ for $k \neq 0$. This means that there is a constant $\gamma$ such that $\phi=\gamma \psi$ on $X_{0} \cap X_{1}$. If $\gamma=0$ it follows that $\phi=0$ also on $\left(X_{0}, X_{1}\right)_{\theta, q} \cap X_{0}$ and the proof can be concluded as in the other cases above. If $\gamma \neq 0$ it follows that $\frac{1}{\gamma} \phi$ is an extension of $\left.\psi\right|_{X_{0} \cap X_{1}}$. Let us check that this extension is continuous on the space $\left(X_{0}, X_{0} \cap X_{1}\right)_{\theta, q}=\left(X_{0}, X_{1}\right)_{\theta, q} \cap X_{0}$. Let $x \in\left(X_{0}, X_{1}\right)_{\theta, q} \cap X_{0}$ and denote by $\alpha$ the norm $x$ in $\left(X_{0}, X_{1}\right)_{\theta, q} \cap X_{0}$. As above, $x=u+s$, where $u=\sum_{k=-\infty}^{-1} 2^{\theta k} x_{k} \in X_{0}, s=\sum_{k=0}^{\infty} 2^{\theta k} x_{k} \in X_{0} \cap X_{1}$, and $\left\|\left(J\left(2^{k}, x_{k} ; X_{0}, X_{1}\right)\right)\right\|_{q} \leq 2 \alpha$. Then

$$
\|u\|_{X_{0}} \leq \sum_{k=-1}^{-\infty} 2^{\theta k} J\left(2^{k}, x_{k} ; X_{0}, X_{1}\right) \leq C_{1} \alpha
$$

and $\|s\|_{X_{0}} \leq\|x\|_{X_{0}}+\|u\|_{X_{0}} \leq\left(C_{1}+1\right) \alpha$. Similarly,

$$
\|s\|_{X_{1}} \leq \sum_{k=0}^{\infty} 2^{(\theta-1) k} J\left(2^{k}, x_{k} ; X_{0}, X_{1}\right) \leq C_{2} \alpha .
$$

Therefore, $\|s\|_{X_{0} \cap X_{1}} \leq C \alpha$. Since $\psi \in\left(X_{0} \cap X_{1}\right)^{*}$, we have

$$
|\phi(x)|=|\psi(s)| \leq C_{3}\|s\|_{X_{0} \cap X_{1}} \leq C_{3} C\|x\|_{\left(X_{0}, X_{1}\right)_{\theta, q} \cap X_{0}},
$$

i.e., $\phi \in\left(\left(X_{0}, X_{1}\right)_{\theta, q} \cap X_{0}\right)^{*}=\left(X_{0}, X_{0} \cap X_{1}\right)_{\theta, q}^{*}$. Hence, $\psi \in\left(X_{0}, X_{0} \cap X_{1}\right)_{\theta, q}^{*}$, which contradicts the assumption that $\psi \notin\left(X_{0}, X_{0} \cap X_{1}\right)_{\theta, q}^{*}$.

The other formulas of the lemma follow from the proved ones by considering the reversed couple.

Remark 3. If $\psi \in\left(X_{0} \cap X_{1}, X_{1}\right)_{\theta, q}^{*}$ and $\psi \in\left(X_{0}, X_{0} \cap X_{1}\right)_{\theta, q}^{*}$, then Lemma 3 implies that $\psi \in\left(X_{0}, X_{1}\right)_{\theta, q}^{*}$ and therefore

$$
\left(X_{0}, X_{1}\right)_{\theta, q, \psi}=\left(X_{0}, X_{1}\right)_{\theta, q} \cap \operatorname{ker} \psi \text {. }
$$


So, in this case

$$
\left(X_{0}, X_{1}\right)_{\theta, q, \psi} \cap X_{0}=\left(X_{0}, X_{1}\right)_{\theta, q, \psi} \cap \operatorname{ker} \psi \cap X_{0},
$$

and we do not get any contradiction.

ExAmple 1. Let $X_{0}=L_{1}(x), X_{1}=L_{1}\left(x^{-1}\right)$ and $\psi(f)=\int_{0}^{\infty} f(s) d s$. It is true that $\left(X_{0}, X_{1}\right)_{\theta, 1}=L_{1}\left(x^{1-2 \theta}\right)$ for $\theta \in(0,1)$. It is not hard to check that $\psi \in\left(X_{0} \cap X_{1}, X_{1}\right)_{\theta, 1}^{*}$ and $\psi \notin\left(X_{0}, X_{0} \cap X_{1}\right)_{\theta, 1}^{*}$ if $0<\theta<1 / 2$, and $\psi \in\left(X_{0}, X_{0} \cap X_{1}\right)_{\theta, 1}^{*}$ and $\psi \notin\left(X_{0} \cap X_{1}, X_{1}\right)_{\theta, 1}^{*}$ if $1 / 2<\theta<1$. On the other hand, we know [17, Theorem 6.1] that $\left(X_{0}, X_{1}\right)_{\theta, 1, \psi}=\left(X_{0}, X_{1}\right)_{\theta, 1}=$ $L_{1}\left(x^{1-2 \theta}\right)$ whenever $\theta \neq 1 / 2$. So, by Lemma 5 ,

$\left(X_{0} \cap X_{1} \cap \operatorname{ker} \psi, X_{1}\right)_{\theta, 1}= \begin{cases}L_{1}\left(x^{1-2 \theta}\right) \cap L_{1}\left(x^{-1}\right) \cap \operatorname{ker} \psi, & 0<\theta<1 / 2, \\ L_{1}\left(x^{1-2 \theta}\right) \cap L_{1}\left(x^{-1}\right), & 1 / 2<\theta<1,\end{cases}$

$\left(X_{0}, X_{0} \cap X_{1} \cap \operatorname{ker} \psi\right)_{\theta, 1}= \begin{cases}L_{1}\left(x^{1-2 \theta}\right) \cap L_{1}(x), & 0<\theta<1 / 2, \\ L_{1}\left(x^{1-2 \theta}\right) \cap L_{1}(x) \cap \operatorname{ker} \psi, & 1 / 2<\theta<1 .\end{cases}$

Lemma 6. Let $\bar{Y}=\left(Y_{0}, Y_{1}\right)$ be a Banach couple, and $\psi \neq 0$ be a bounded linear functional on $Y_{0} \cap Y_{1}$.

(i) If $\psi \in Y_{0}^{*}$ and $\psi \in Y_{1}^{*}$, then

$$
Y_{0} \cap \operatorname{ker} \psi+Y_{1} \cap \operatorname{ker} \psi=\left(Y_{0}+Y_{1}\right) \cap \operatorname{ker} \psi \text {. }
$$

(ii) If $\psi \in Y_{1}^{*}$, then

$$
Y_{0}+Y_{1} \cap \operatorname{ker} \psi=Y_{0}+Y_{1}
$$

(iii) If $\psi \in Y_{0}^{*}$, then

$$
Y_{0} \cap \operatorname{ker} \psi+Y_{1}=Y_{0}+Y_{1} .
$$

Proof. We will only prove the first point because the others are proved in the same way. Without loss of generality we can assume that $\|\psi\|_{\left(Y_{0} \cap Y_{1}\right)^{*}} \leq 1$. It is clear that

$$
Y_{0} \cap \operatorname{ker} \psi+Y_{1} \cap \operatorname{ker} \psi \subset\left(Y_{0}+Y_{1}\right) \cap \operatorname{ker} \psi .
$$

If $y \in\left(Y_{0}+Y_{1}\right) \cap \operatorname{ker} \psi$, there are $y_{0} \in Y_{0}$ and $y_{1} \in Y_{1}$ such that $y_{0}+y_{1}=y$ and $\left\|y_{0}\right\|_{Y_{0}}+\left\|y_{1}\right\|_{Y_{1}} \leq 2\|y\|_{Y_{0}+Y_{1}}$. Since $\psi(y)=0$ we have $\psi\left(y_{0}\right)+\psi\left(y_{1}\right)=0$. We can find $w \in Y_{0} \cap Y_{1}$ such that $\psi(w)=1$ and $\max \left(\|w\|_{Y_{0}},\|w\|_{Y_{1}}\right) \leq 2$. Let $\widetilde{y}_{0}=y_{0}-\psi\left(y_{0}\right) w$ and $\widetilde{y}_{1}=y_{1}+\psi\left(y_{0}\right) w=y_{1}-\psi\left(y_{1}\right) w$. Then $y=\widetilde{y}_{0}+\widetilde{y}_{1}$ and

$$
\begin{aligned}
\left\|\widetilde{y}_{0}\right\|_{Y_{0}}+\left\|\widetilde{y}_{1}\right\|_{Y_{1}} & \leq\left\|y_{0}\right\|_{Y_{0}}+\left|\psi\left(y_{0}\right)\right|\|w\|_{Y_{0}}+\left\|y_{1}\right\|_{Y_{1}}+\left|-\psi\left(y_{1}\right)\right|\|w\|_{Y_{1}} \\
& \leq 3\left(\left\|y_{0}\right\|_{Y_{0}}+\left\|y_{1}\right\|_{Y_{1}}\right) \leq 6\|y\|_{Y_{0}+Y_{1}} \text {. }
\end{aligned}
$$

Proof of Theorem 3. First, we recall the following statements (see [3, Lemma 5 and Corollary 1] or [17, Property 2]): (I) if $\left(X_{0}, X_{1}\right)_{\theta, q, \psi}$ is closed in $\left(X_{0}, X_{1}\right)_{\theta, q}$, then either $\left(X_{0}, X_{1}\right)_{\theta, q, \psi}=\left(X_{0}, X_{1}\right)_{\theta, q} \cap \operatorname{ker} \psi$ (if $\left.\psi \in\left(X_{0}, X_{1}\right)_{\theta, q}^{*}\right)$ or $\left(X_{0}, X_{1}\right)_{\theta, q, \psi}=\left(X_{0}, X_{1}\right)_{\theta, q} \quad\left(\right.$ if $\left.\psi \notin\left(X_{0}, X_{1}\right)_{\theta, q}^{*}\right)$; 
(II) $\left(X_{0}, X_{1}\right)_{\theta, q, \psi}=\left(X_{0}, X_{1}\right)_{\theta, q} \cap \operatorname{ker} \psi$ if and only if $\psi \in\left(X_{0}, X_{1}\right)_{\theta, q}^{*}$. Moreover, it follows easily that

$$
\begin{aligned}
& \left(X_{0}, X_{0} \cap X_{1} \cap \operatorname{ker} \psi\right)_{\theta, q}=\left(X_{0}, X_{0} \cap X_{1}\right)_{\theta, q, \psi}, \\
& \left(X_{0} \cap X_{1} \cap \operatorname{ker} \psi, X_{1}\right)_{\theta, q}=\left(X_{0} \cap X_{1}, X_{1}\right)_{\theta, q, \psi} .
\end{aligned}
$$

Therefore, the "if and only if" statement at the beginning of the theorem is a consequence of (i) and (ii). So, it is sufficient to prove (i) and (ii).

(i) Assume that $\left(X_{0}, X_{1}\right)_{\theta, q, \psi}=\left(X_{0}, X_{1}\right)_{\theta, q} \cap \operatorname{ker} \psi$. Then $\psi \in\left(X_{0}, X_{1}\right)_{\theta, q}^{*}$ and Lemmas 4 and 5 imply that

$$
\begin{aligned}
\left(X_{0}, X_{0} \cap X_{1} \cap \operatorname{ker} \psi\right)_{\theta, q} & =\left(X_{0}, X_{1}\right)_{\theta, q, \psi} \cap X_{0} \cap \operatorname{ker} \psi \\
& =\left(X_{0}, X_{1}\right)_{\theta, q} \cap X_{0} \cap \operatorname{ker} \psi \\
& =\left(X_{0}, X_{0} \cap X_{1}\right)_{\theta, q} \cap \operatorname{ker} \psi
\end{aligned}
$$

and similarly

$$
\begin{aligned}
\left(X_{0} \cap X_{1} \cap \operatorname{ker} \psi, X_{1}\right)_{\theta, q} & =\left(X_{0}, X_{1}\right)_{\theta, q, \psi} \cap X_{1} \cap \operatorname{ker} \psi \\
& =\left(X_{0}, X_{1}\right)_{\theta, q} \cap X_{1} \cap \operatorname{ker} \psi \\
& =\left(X_{0} \cap X_{1}, X_{1}\right)_{\theta, q} \cap \operatorname{ker} \psi .
\end{aligned}
$$

Conversely, assume that

$$
\begin{aligned}
& \left(X_{0}, X_{0} \cap X_{1} \cap \operatorname{ker} \psi\right)_{\theta, q}=\left(X_{0}, X_{0} \cap X_{1}\right)_{\theta, q} \cap \operatorname{ker} \psi, \\
& \left(X_{0} \cap X_{1} \cap \operatorname{ker} \psi, X_{1}\right)_{\theta, q}=\left(X_{0} \cap X_{1}, X_{1}\right)_{\theta, q} \cap \operatorname{ker} \psi .
\end{aligned}
$$

Then $\psi \in\left(X_{0}, X_{0} \cap X_{1}\right)_{\theta, q}^{*}$ and $\psi \in\left(X_{0} \cap X_{1}, X_{1}\right)_{\theta, q}^{*}$. Therefore, by Lemmas 3 and $4, \psi \in\left(X_{0}, X_{1}\right)_{\theta, q}^{*}$, which implies that

$$
\left(X_{0}, X_{1}\right)_{\theta, q, \psi}=\left(X_{0}, X_{1}\right)_{\theta, q} \cap \operatorname{ker} \psi .
$$

(ii) Let $\left(X_{0}, X_{1}\right)_{\theta, q, \psi}=\left(X_{0}, X_{1}\right)_{\theta, q}$. If (a) $\psi \in\left(X_{0}, X_{0} \cap X_{1}\right)_{\theta, q}^{*}$ and $\psi \notin\left(X_{0} \cap X_{1}, X_{0}\right)_{\theta, q}^{*}$, then, by Lemmas 4 and 5 ,

$$
\begin{aligned}
\left(X_{0}, X_{0} \cap X_{1} \cap \operatorname{ker} \psi\right)_{\theta, q} & =\left(X_{0}, X_{1}\right)_{\theta, q, \psi} \cap X_{0} \cap \operatorname{ker} \psi \\
& =\left(X_{0}, X_{1}\right)_{\theta, q} \cap X_{0} \cap \operatorname{ker} \psi \\
& =\left(X_{0}, X_{0} \cap X_{1}\right)_{\theta, q} \cap \operatorname{ker} \psi \\
\left(X_{0} \cap X_{1} \cap \operatorname{ker} \psi, X_{1}\right)_{\theta, q} & =\left(X_{0}, X_{1}\right)_{\theta, q, \psi} \cap X_{1} \\
& =\left(X_{0}, X_{1}\right)_{\theta, q} \cap X_{1}=\left(X_{0} \cap X_{1}, X_{1}\right)_{\theta, q} .
\end{aligned}
$$

In the cases (b) $\psi \notin\left(X_{0}, X_{0} \cap X_{1}\right)_{\theta, q}^{*}$ and $\psi \in\left(X_{0} \cap X_{1}, X_{0}\right)_{\theta, q}^{*}$ and (c) $\psi \notin\left(X_{0}, X_{0} \cap X_{1}\right)_{\theta, q}^{*}$ and $\psi \notin\left(X_{0} \cap X_{1}, X_{0}\right)_{\theta, q}^{*}$ we end up in a very similar manner. If $\psi \in\left(X_{0}, X_{0} \cap X_{1}\right)_{\theta, q}^{*}$ and $\psi \in\left(X_{0} \cap X_{1}, X_{0}\right)_{\theta, q}^{*}$ then again $\psi \in$ $\left(X_{0}, X_{1}\right)_{\theta, q}^{*}$ and $\left(X_{0}, X_{1}\right)_{\theta, q, \psi} \subset\left(X_{0}, X_{1}\right)_{\theta, q} \cap$ ker $\psi$, which contradicts the assumption that $\left(X_{0}, X_{1}\right)_{\theta, q, \psi}=\left(X_{0}, X_{1}\right)_{\theta, q}$.

Conversely, assume, for example, that both

$$
\left(X_{0}, X_{0} \cap X_{1} \cap \operatorname{ker} \psi\right)_{\theta, q}=\left(X_{0}, X_{0} \cap X_{1}\right)_{\theta, q} \cap \operatorname{ker} \psi
$$


and

$$
\left(X_{0} \cap X_{1} \cap \operatorname{ker} \psi, X_{1}\right)_{\theta, q}=\left(X_{0} \cap X_{1}, X_{1}\right)_{\theta, q} .
$$

Then condition (a) is true. So, by Lemmas 3-6 with $Y_{0}=\left(X_{0}, X_{0} \cap X_{1}\right)_{\theta, q}$ and $Y_{1}=\left(X_{0} \cap X_{1}, X_{1}\right)_{\theta, q}$, we have

$$
\begin{aligned}
\left(X_{0}, X_{1}\right)_{\theta, q} & =\left(X_{0}, X_{1}\right)_{\theta, q} \cap X_{0}+\left(X_{0}, X_{1}\right)_{\theta, q} \cap X_{1} \\
& =\left(X_{0}, X_{0} \cap X_{1}\right)_{\theta, q}+\left(X_{0} \cap X_{1}, X_{1}\right)_{\theta, q} \\
& =\left(X_{0}, X_{0} \cap X_{1}\right)_{\theta, q} \cap \operatorname{ker} \psi+\left(X_{0} \cap X_{1}, X_{1}\right)_{\theta, q} \\
& =\left(X_{0}, X_{0} \cap X_{1} \cap \operatorname{ker} \psi\right)_{\theta, q}+\left(X_{0} \cap X_{1} \cap \operatorname{ker} \psi, X_{1}\right)_{\theta, q} .
\end{aligned}
$$

Using Lemma 6 for the second time for the spaces $Y_{0}=\left(X_{0}, X_{1}\right)_{\theta, q, \psi} \cap X_{0}$ and $Y_{1}=\left(X_{0}, X_{1}\right)_{\theta, q, \psi} \cap X_{1}$ shows that the last expression is equal to

$$
\begin{aligned}
\left(X_{0}, X_{1}\right)_{\theta, q, \psi} \cap X_{0} \cap \operatorname{ker} \psi & +\left(X_{0}, X_{1}\right)_{\theta, q, \psi} \cap X_{1} \\
= & \left(X_{0}, X_{1}\right)_{\theta, q, \psi} \cap X_{0}+\left(X_{0}, X_{1}\right)_{\theta, q, \psi} \cap X_{1}=\left(X_{0}, X_{1}\right)_{\theta, q, \psi} .
\end{aligned}
$$

The other two cases are considered in the same way.

We now have everything we need to prove Theorem 2 (equivalently, Theorem 1).

Proof of Theorem 2. The special cases when $\psi$ is bounded on one of the endpoint spaces were proved by Ivanov and Kalton [6]. If $\psi \in X_{0}^{*}$ it follows that $\alpha_{\infty}=\beta_{\infty}=\alpha=0$ and therefore we have a closed subspace when $\theta>\beta=\beta_{0}$ and when $\theta<\alpha_{0}$ and for no other $\theta$. That subspace has codimension one in the latter case and zero in the former. If instead $\psi \in X_{1}^{*}$, it follows that $\alpha_{0}=\beta_{0}=\beta=1$ and we have a closed subspace that equals the whole space if $\theta<\alpha=\alpha_{\infty}$ and a closed subspace of codimension one if $\theta>\beta_{\infty}$. The subspace is not closed for any other $\theta$. The rest of Theorem 2 follows from that result, Theorem 3, and Lemmas 1 and 2.

\section{Inter polation of intersections of weighted $L_{p}$-spaces generated} by the integral functional. Let $w(x)$ be a positive measurable function on $(0, \infty), 1 \leq p \leq \infty$. As usual, we define $L_{p}(w)$ as the space of all measurable functions $f:(0, \infty) \rightarrow \mathbb{R}$ with the norm

$$
\|f\|_{w, p}=\left(\int_{0}^{\infty}|f(x)|^{p} w(x) d x\right)^{1 / p}
$$

(with the usual modification for $p=\infty$ ). Let us consider the linear functional

$$
\phi(f)=\int_{0}^{\infty} f(x) d x
$$

with the domain $D$ consisting of all measurable functions $f:(0, \infty) \rightarrow \mathbb{R}$ such that $\int_{a}^{b}|f(x)| d x<\infty$ for all $0<a<b<\infty$ and $\lim _{a \rightarrow 0, b \rightarrow \infty} \int_{a}^{b} f(x) d x$ exists. Denote its kernel by $N$. 
It might seem restrictive to choose a particular linear functional but actually it is sufficient for understanding the corresponding situation for a larger class of positive linear functionals. If we used a linear functional of the form $\psi(f)=\int_{0}^{\infty} f(x) g(x) d x$ where $g$ is bounded and strictly positive, we could perform a change of measure by considering the measure $g(x) d x$ and we end up in a situation with the standard integral functional and weights $w_{i} / g, i=0,1$. The $K$-functional of $\psi$ in the dual couple of the couple with weights $w_{i}, i=0,1$, is equal to the $K$-functional of the standard integral functional $\phi$ in the dual couple of the couple with weights $w_{i} / g$, and the answer to the interpolation of intersections question only depends on that $K$-functional.

Assume that the restriction of the standard integral functional $\phi$ to the intersection $L_{p_{0}}\left(w_{0}\right) \cap L_{p_{1}}\left(w_{1}\right)$ is bounded. Using Theorem 1 and arguing as in [3], we can find necessary and sufficient conditions for equality (4) to hold (without any restrictions on the dilation indices of the Peetre $K$-functional of the integral functional $\phi$ in the couple $\left.\left(L_{p_{0}}\left(w_{0}\right)^{*}, L_{p_{1}}\left(w_{1}\right)^{*}\right)\right)$. We state here only a result in the case when $p_{0}=p_{1}=1$. Let $w(x)=w_{0}(x) / w_{1}(x)$ and let $w^{-1}(x)$ be the function inverse to $w(x)$.

THEOREM 4. Suppose that weight functions $w_{0}(x)$ and $w_{1}(x)$ satisfy the following conditions:

- $w_{0}(x)$ is increasing,

- $w_{1}(x)$ is decreasing,

- $\lim _{x \rightarrow 0} w(x)=\lim _{x \rightarrow \infty} 1 / w(x)=0$.

Let $\alpha, \beta, \alpha_{0}, \beta_{0}, \alpha_{\infty}$, and $\beta_{\infty}$ be the dilation indices of the function $k(t)=$ $1 / w_{0}\left(w^{-1}(1 / t)\right)$. Then the formula

$$
\left(L_{1}\left(w_{0}\right) \cap N, L_{1}\left(w_{1}\right) \cap N\right)_{\theta, q}=\left(L_{1}\left(w_{0}\right), L_{1}\left(w_{1}\right)\right)_{\theta, q} \cap N
$$

is equivalent to condition (7).

Proof. Since the restriction of $\phi$ to the intersection $L_{1}\left(w_{0}\right) \cap L_{1}\left(w_{1}\right)$ is a bounded functional, the necessity of condition (7) follows at once from Theorem 1 and [3, Corollary 2].

Conversely, assume that (7) is satisfied. By Theorem 2, it is sufficient to prove that

$$
\left(L_{1}\left(w_{0}\right), L_{1}\left(w_{1}\right)\right)_{\theta, q} \cap N \subset L_{1}\left(w_{0}\right) \cap N+L_{1}\left(w_{1}\right) \cap N .
$$

For every $f \in\left(L_{1}\left(w_{0}\right), L_{1}\left(w_{1}\right)\right)_{\theta, q} \cap N$ consider the representation $f=f_{0}+f_{1}$, where $f_{0}=f \cdot \chi_{(0,1]}, f_{1}=f-f_{0}$. The conditions on the weights $w_{0}$ and $w_{1}$ imply that $f_{0} \in L_{1}\left(w_{0}\right)$ and $f_{1} \in L_{1}\left(w_{1}\right)$. Moreover, it is not hard to check that $f_{0}$ and $f_{1}$ belong to the domain $D$ of the functional $\phi$. Let $g \in L_{1}\left(w_{0}\right) \cap L_{1}\left(w_{1}\right)$ be such that $\phi(g)=1$ and let $g_{0}=f_{0}-\phi\left(f_{0}\right) g$ and 
$g_{1}=f_{0}+\phi\left(f_{0}\right) g$. Then $f=g_{0}+g_{1}$ and $g_{i} \in L_{1}\left(w_{i}\right) \cap N(i=0,1)$. So, inclusion (10) is proved, and hence we have (9).

Complementing Corollary 7 from [3], we shall show that for arbitrary numbers $a, b, c$, and $d$ with $0 \leq a \leq \min (b, c) \leq \max (b, c) \leq d \leq 1$ there exist weight functions $w_{0}(x)$ and $w_{1}(x)$ such that the dilation indices $\alpha, \beta_{0}$, $\alpha_{\infty}$, and $\beta$ of the function $k(t)=1 / w_{0}\left(w^{-1}(1 / t)\right)$ coincide with $a, b, c$, and $d$, respectively.

Following [3], we take a two-sided increasing sequence $\left(x_{k}\right)_{k=-\infty}^{\infty}$ of positive numbers such that $x_{0}=1$ and $\lim _{|k| \rightarrow \infty} x_{k+1} / x_{k}=\infty$.

If $b>0$, we define a function $\nu^{\prime}$ in the following way: $\nu^{\prime}(1)=1$,

$$
\nu^{\prime}(x)= \begin{cases}\left(x / x_{2 k}\right)^{c} \nu^{\prime}\left(x_{2 k}\right), & x_{2 k} \leq x \leq x_{2 k+1}, \\ \left(x / x_{2 k+1}\right)^{d} \nu^{\prime}\left(x_{2 k+1}\right), & x_{2 k+1} \leq x \leq x_{2 k+2},\end{cases}
$$

for $k=0,1,2, \ldots$, and

$$
\nu^{\prime}(x)= \begin{cases}\left(x / x_{2 k}\right)^{a} \nu^{\prime}\left(x_{2 k}\right), & x_{2 k-1} \leq x \leq x_{2 k}, \\ \left(x / x_{2 k-1}\right)^{b} \nu^{\prime}\left(x_{2 k-1}\right), & x_{2 k-2} \leq x \leq x_{2 k-1},\end{cases}
$$

for $k=0,-1,-2, \ldots$

If $b=0$ and $d>0$, we define $\nu^{\prime}(x)$ as before if $x \in[1, \infty)$, and for $x \in(0,1]$ we put $\nu^{\prime}(x)=1 / \ln (e / x)$.

Finally, if $d=0$, we put $\nu^{\prime}(x)=1 / \ln (e / x)$ for $x \in(0,1]$ and $\nu^{\prime}(x)=$ $\ln (e x)$ for $x \in[1, \infty)$.

It can easily be checked that $\nu^{\prime}(x)$ is increasing, $\nu^{\prime}(x) / x$ is decreasing, and the range of $\nu^{\prime}(x)$ is $(0, \infty)$. There exists an increasing concave function $\nu(x)$ such that $\nu^{\prime}(x) \leq \nu(x) \leq 2 \nu^{\prime}(x)(x>0)$ (see [10, Theorem 2.1.1]). The definition of $\nu^{\prime}$ readily implies that its dilation indices $\alpha, \beta_{0}, \alpha_{\infty}$, and $\beta$ (therefore, also those of $\nu$ ) coincide with $a, b, c$, and $d$, respectively.

We define $w_{0}(x)=x$ and $w_{1}(x)=x \nu^{-1}(1 / x)\left(\nu^{-1}\right.$ is the inverse function to $\nu)$. Since these functions satisfy the conditions of Theorem 4 and $\nu(x)=$ $1 / w_{0}\left(w^{-1}(1 / t)\right)$, applying this theorem we arrive at the following result: if the weight functions $w_{0}$ and $w_{1}$ are defined as earlier, then equality (9) holds if and only if $\theta \in(0, a) \cup(b, c) \cup(d, 1)$.

6. The shift operator and its spectrum on weighted $\ell_{q}$ spaces. In $[2,3,17]$, weaker versions of Theorems 1 and 2 were proved using an approach developed by Ivanov and Kalton in [6] for comparison of the interpolation spaces $\left(X_{0}, X_{1}\right)_{\theta, q}$ and $\left(N_{0}, X_{1}\right)_{\theta, q}$, where $\psi \in X_{0}^{*}$ and $N_{0}=\operatorname{ker} \psi$. The key idea of that approach is to reduce the general interpolation problem for subspaces to the study of the shift operator on a certain weighted $\ell_{p}$-space. Note that the theorems obtained here imply the interpolation results of [6] (see also [3] and [17]). In the remainder of this article we will work in the 
opposite direction and use our interpolation results to derive more general results concerning the shift operator and its spectrum on weighted $\ell_{p}$-spaces.

We start with some notation and preliminary remarks. Putting

$$
\mu_{n}=\left(k\left(2^{-n}\right)\right)^{-1} \quad(n \in \mathbb{Z})
$$

we see that $\mu_{n}>0$, and since $k(t)=K\left(t, \psi ; X_{0}^{*}, X_{1}^{*}\right)$ is an increasing and concave function on $(0, \infty)$, we have

$$
\mu_{n} \leq \mu_{n+1} \leq 2 \mu_{n} \quad(n \in \mathbb{Z})
$$

Furthermore, it is easy to check that

$$
\begin{aligned}
\alpha & =-\lim _{n \rightarrow \infty} \frac{1}{n} \log _{2} \sup _{k \in \mathbb{Z}} \frac{\mu_{k}}{\mu_{n+k}}, & \beta & =\lim _{n \rightarrow \infty} \frac{1}{n} \log _{2} \sup _{k \in \mathbb{Z}} \frac{\mu_{k}}{\mu_{k-n}}, \\
\alpha_{\infty} & =-\lim _{n \rightarrow \infty} \frac{1}{n} \log _{2} \sup _{k \leq 0} \frac{\mu_{k-n}}{\mu_{k}}, & \beta_{\infty} & =\lim _{n \rightarrow \infty} \frac{1}{n} \log _{2} \sup _{k \leq 0} \frac{\mu_{k}}{\mu_{k-n}}, \\
\alpha_{0} & =-\lim _{n \rightarrow \infty} \frac{1}{n} \log _{2} \sup _{k \geq 0} \frac{\mu_{k}}{\mu_{n+k}}, & \beta_{0} & =\lim _{n \rightarrow \infty} \frac{1}{n} \log _{2} \sup _{k \geq 0} \frac{\mu_{k+n}}{\mu_{k}} .
\end{aligned}
$$

Let $\ell_{q}(\mu)$ be the space of two-sided numerical sequences $a=\left(a_{k}\right)_{k=-\infty}^{\infty}$ with the norm

$$
\|a\|_{q, \mu}=\left\{\sum_{k \in \mathbb{Z}}\left|a_{k}\right|^{q} \mu_{k}^{q}\right\}^{1 / q} .
$$

In what follows, we shall consider the shift operator $S\left(\left(a_{k}\right)\right)=\left(a_{k-1}\right)$, its inverse $S^{-1}$, and also the operators $T_{\theta}=S-2^{\theta} I(0<\theta<1)$, where $I$ is the identity mapping. By (12), both $S$ and $S^{-1}$ are bounded on $\ell_{q}(\mu)$, and $\|S\| \leq 2,\left\|S^{-1}\right\| \leq 1$.

REMARK 4. If $r(S)$ denotes the spectral radius of the shift operator, then $2^{\alpha}=1 / r\left(S^{-1}\right)$ and $2^{\beta}=r(S)$. Therefore, it is clear that $T_{\theta}$ is invertible if $\theta<\alpha$ or $\theta>\beta$.

The proof of Theorem 1 from [3] (see also [17, Theorems 4.2 and 4.3]) shows that the following proposition is a consequence of Theorems 1 and 2 .

Proposition 1. Let $0<\theta<1$ and $1 \leq q<\infty$. The operator $T_{\theta}$ is closed in $\ell_{q}(\mu)$ if and only if

$$
\theta \in(0, \alpha) \cup\left(\beta_{\infty}, \alpha_{0}\right) \cup\left(\beta_{0}, \alpha_{\infty}\right) \cup(\beta, 1) .
$$

Moreover, if $\theta \in(0, \alpha) \cup\left(\beta_{0}, \alpha_{\infty}\right) \cup(\beta, 1)$, then $\operatorname{Im} T_{\theta}=\ell_{q}(\mu)$; if $\theta \in\left(\beta_{\infty}, \alpha_{0}\right)$, then $\operatorname{Im} T_{\theta}$ is a closed subspace of $\ell_{q}(\mu)$ of codimension 1 consisting of all $\left(a_{k}\right)_{k \in \mathbb{Z}} \in \ell_{q}(\mu)$ such that

$$
\sum_{k \in \mathbb{Z}} 2^{k \theta} a_{k}=0 .
$$

The weight $\mu=\left(\mu_{n}\right)_{n \in \mathbb{Z}}$ from the last proposition satisfies the condition: there are a couple $\left(X_{0}, X_{1}\right)$ and a linear functional $\psi \in\left(X_{0} \cap X_{1}\right)^{*}$ such 
that for the $K$-functional $k(t)=K\left(t, \psi ; X_{0}^{*}, X_{1}^{*}\right)$ we have (11). Next, we will check that assumption (12) is not only necessary for the existence of such a linear functional but also sufficient. Consider the couple $\left(\ell_{1}, \ell_{1}\left(2^{k}\right)\right)$ and linear functionals of the form $\psi(x)=\sum v_{k} x_{k}$, where $0<v_{k} \leq v_{k+1} \leq 2 v_{k}$. The last condition implies that the linear functional $\psi$ is bounded on the intersection $\ell_{1} \cap \ell_{1}\left(2^{k}\right)$. The $K$-functional $k$ for such a linear functional has the property that $k\left(2^{n}\right)=v_{n}(n \in \mathbb{Z})$. Thus, the functional we are looking for is defined by letting $v_{k}=1 / \mu_{-k}$.

TheOREM 5. Let $0<\theta<1,1 \leq q<\infty$ and let $\left(\mu_{n}\right)_{n \in \mathbb{Z}}$ be a positive sequence such that $\mu_{n} \leq \mu_{n+1} \leq 2 \mu_{n}$ for all $n$. The operator $T_{\theta}$ is closed in $\ell_{q}(\mu)$ if and only if

$$
\theta \in(0, \alpha) \cup\left(\beta_{\infty}, \alpha_{0}\right) \cup\left(\beta_{0}, \alpha_{\infty}\right) \cup(\beta, 1) .
$$

Moreover, if $\theta \in(0, \alpha) \cup\left(\beta_{0}, \alpha_{\infty}\right) \cup(\beta, 1)$, then $\operatorname{Im} T_{\theta}=\ell_{q}(\mu)$; if $\theta \in\left(\beta_{\infty}, \alpha_{0}\right)$, then $\operatorname{Im} T_{\theta}$ is a closed subspace of $\ell_{q}(\mu)$ of codimension 1 consisting of all $\left(a_{k}\right)_{k \in \mathbb{Z}} \in \ell_{q}(\mu)$ such that

$$
\sum_{k \in \mathbb{Z}} 2^{k \theta} a_{k}=0
$$

If we want to know when $T_{\theta}$ is invertible we also have to know if it is injective or not in the cases when we know that it is surjective, i.e., when $\theta \in(0, \alpha) \cup\left(\beta_{0}, \alpha_{\infty}\right) \cup(\beta, 1)$. When $\theta \in(0, \alpha) \cup(\beta, 1)$ it is shown in [3] and [17] that $T_{\theta}$ is injective; we will prove that in the remaining cases it is not.

Proposition 2. If $\theta \in\left(\beta_{0}, \alpha_{\infty}\right)$, then $T_{\theta}$ is not injective.

Proof. It is sufficient to prove that $\left(2^{-\theta k}\right) \in \ell_{q}(\mu)$ since that element then lies in the kernel of $T_{\theta}$. If $\varepsilon>0$ is sufficiently small, then from the inequality $\theta>\alpha_{0}$ and from the definition of the index $\alpha_{0}$ it follows that

whence

$$
\sup _{n \geq 0} \frac{\mu_{n}}{\mu_{k+n}} \geq c_{1} 2^{-k(\theta-\varepsilon)}, \quad k=1,2, \ldots,
$$

$$
\mu_{k} 2^{-\theta k} \leq \frac{\mu_{0}}{c_{1}} 2^{-\varepsilon k}, \quad k=1,2, \ldots
$$

Similarly, since $\theta<\beta_{\infty}$, for sufficiently small $\eta>0$ we have

$$
\mu_{-k} 2^{\theta k} \leq \frac{\mu_{0}}{c_{2}} 2^{-\eta k}, \quad k=1,2, \ldots,
$$

and therefore

$$
\sum_{k \in \mathbb{Z}} 2^{-k \theta q} \mu_{k}^{q}<\infty
$$

Since $\alpha_{0} \leq \beta_{0}$ and $\alpha_{\infty} \leq \beta_{\infty}$, the proof is complete.

COROLlaRY 1. The operator $T_{\theta}$ is invertible if and only if $\theta<\alpha$ or $\theta>\beta$. 
THEOREM 6. The spectrum of $S$ on $\ell_{q}(\mu)$ is the set $\left\{z \in \mathbb{C}\left|2^{\alpha} \leq\right| z \mid \leq 2^{\beta}\right\}$.

Proof. The spectrum is invariant under rotation since the operator $V_{\lambda}\left(\left(\alpha_{k}\right)\right)=\left(\lambda^{k} \alpha_{k}\right)$ is an isometry for $\lambda$ on the unit circle in $\mathbb{C}$. Therefore, the theorem follows from Corollary 1.

It is worth noting that the Stein-Weiss Theorem [5, Theorem 5.4.4] implies that the space $\ell_{p}(\mu)$ is an interpolation space of the couple $\left(\ell_{p}, \ell_{p}\left(2^{k}\right)\right)$ if $\mu$ satisfies condition (12). The operator $T_{\theta}$ is invertible on $\ell_{p}$ if $\theta \neq 0$ and on $\ell_{p}\left(2^{k}\right)$ if $\theta \neq 1$. If we could "interpolate" the inverse of $T_{\theta}$ for a certain $\theta \in(0,1)$ to the space $\ell_{p}(\mu)$, we would know that $T_{\theta}$ is surjective on that space. There is, however, a problem when we try to perform that interpolation: the inverse operators on the endpoint spaces do not have to agree on the whole of the intersection. In other words, the operator $T_{\theta}$ is not an injection as an operator defined on the sum $\ell_{p}+\ell_{p}\left(2^{k}\right)$, that is, the inverse of $T_{\theta}$ (on this sum) does not exist (see [4, Remark 3.1] and references therein). To be able to formulate results about the spectrum of operators on interpolation spaces, authors have usually imposed the assumption that the inverses agree, see e.g. [16]. In our particular case we have two possible inverses,

$$
\begin{aligned}
& U_{\theta}^{+}\left(\left(\alpha_{k}\right)\right)=\left(2^{-(n+1) \theta} \sum_{n+1}^{\infty} 2^{\theta k} \alpha_{k}\right)_{n}, \\
& U_{\theta}^{-}\left(\left(\alpha_{k}\right)\right)=\left(-2^{-(n+1) \theta} \sum_{-\infty}^{n} 2^{\theta k} \alpha_{k}\right)_{n},
\end{aligned}
$$

and they agree only on $\left(\alpha_{k}\right)$ with $\sum 2^{\theta k} \alpha_{k}=0$. This is actually precisely the same kind of situation that was considered by Krugljak, Maligranda and Persson in [11] when they interpolated Hardy-type operators. The operators $U_{\theta}^{+}$and $U_{\theta}^{-}$can be seen as discrete Hardy-type averaging operators. They can be converted to the usual Hardy operator forms

$$
\frac{1}{x} \int_{0}^{x} f(s) d s \text { and }-\frac{1}{x} \int_{x}^{\infty} f(s) d s
$$

by letting $f(s)=\alpha_{k}$ for $2^{\theta k} \leq s<2^{\theta(k+1)}$ and $x=2^{\theta(k+1)}$. We see that $U_{\theta}^{-}$ is bounded on $\ell_{p}$ for $\theta>0$ and $U_{\theta}^{+}$on $\ell_{p}\left(2^{n}\right)$ for $\theta<1$.

\section{References}

[1] S. V. Astashkin, About interpolation of subspaces of rearrangement invariant spaces generated by Rademacher system, Int. J. Math. Math. Sci. 25 (2001), 451-465.

[2] - Interpolation of intersections generated by a linear functional, Funktsional. Anal. i Prilozhen. 39 (2005), no. 2, 61-64 (in Russian); English transl.: Funct. Anal. Appl. 39 (2005), 131-134. 
[3] S. V. Astashkin, Interpolation of intersections by the real method, Algebra i Analiz 17 (2005), no. 2, 33-69 (in Russian); English transl.: St. Petersburg Math. J. 17 (2006), 239-266.

[4] -, Images of operators in rearrangement invariant spaces and interpolation, in: Function Spaces (Wrocław, 2001), World Sci., River Edge, NJ, 2003, 49-64.

[5] J. Bergh and J. Löfström, Interpolation Spaces. An Introduction, Grundlehren Math. Wiss. 223, Springer, Berlin, 1976.

[6] S. Ivanov and N. Kalton, Interpolation of subspaces and applications to exponential bases, Algebra i Analiz 13 (2001), no. 2, 93-115 (in Russian); English transl.: St. Petersburg Math. J. 13 (2002), 221-239.

[7] S. Janson, Interpolation of subcouples and quotient couples, Ark. Mat. 31 (1993), 307-338.

[8] S. Kaijser and P. Sunehag, Interpolation subspaces and the unit problem, in: Function Spaces, Interpolation Theory and Related Topics (Lund, 2000), de Gruyter, Berlin, $2002,345-353$.

[9] S. V. Kislyakov and Q. H. Xu, Real interpolation and singular integrals, Algebra $\mathrm{i}$ Analiz 8 (1996), no. 4, 75-109 (in Russian); English transl.: St. Petersburg Math. J. 8 (1997), 593-615.

[10] S. G. Kreǐn, Yu. I. Petunin and E. M. Semënov, Interpolation of Linear Operators, Nauka, Moscow, 1978 (in Russian); English transl.: Transl. Math. Monogr. 54, Amer. Math. Soc., Providence, RI, 1982.

[11] N. Krugljak, L. Maligranda, and L.-E. Persson, The failure of the Hardy inequality and interpolation of intersections, Ark. Mat. 37 (1999), 323-344.

[12] J.-L. Lions et E. Magenes, Problèmes aux limites non homogènes et applications, Vols. 1-2, Dunod, Paris, 1968.

[13] J. Löfström, Interpolation of subspaces, Techn. Rep. no. 10, Univ. of Göteborg, 1997, $63 \mathrm{pp}$.

[14] L. Maligranda, On commutativity of interpolation with intersection, Rend. Circ. Mat. Palermo (2) Suppl. 10 (1985), 113-118.

[15] G. Pisier, Interpolation between $H^{p}$ spaces and noncommutative generalizations. I, Pacific J. Math. 155 (1992), 341-368.

[16] J. D. Stafney, Set approximation by lemniscates and the spectrum of an operator on an interpolation space, ibid. 60 (1975), 253-265.

[17] P. Sunehag, Subcouples of codimension one and interpolation of operators that almost agree, J. Approx. Theory 130 (2004), 78-98.

[18] H. Triebel, Eine Bemerkung zur nicht-kommutativen Interpolation, Math. Nachr. 69 (1975), 57-60.

[19] R. Wallstén, Remarks on interpolation of subspaces, in: Function Spaces and Applications (Lund, 1986), Lecture Notes in Math. 1302, Springer, Berlin, 1988, 410-419.

Department of Mathematics and Mechanics

NICTA

Samara State University

Acad. Pavlov Street

443011 Samara, Russia

E-mail: astashkn@ssu.samara.ru
Locked Bag 8001

Canberra, 2601 ACT, Australia

E-mail: peter.sunehag@nicta.com.au 